Société d'histoire de la révolution de 1848 et des

révolutions du XIXe siècle

49 | 2014

1814-1815. Expériences de la discontinuité

\title{
1814-1815 : construction dramatique des événements et modes théâtraux de symbolisation du présent
}

1814-5: the dramatic construction of events and the theatrical ways of symbolizing the present

Dramatische Konstruktion von Ereignissen und die Darstellung symbolischer Gegenwart im Theater (1814-15)

\section{Olivier Bara}

\section{CpenEdition}

\section{Journals}

Édition électronique

URL : http://journals.openedition.org/rh19/4750

DOI : $10.4000 /$ rh 19.4750

ISSN : $1777-5329$

Éditeur

La Société de 1848

Édition imprimée

Date de publication : 1 décembre 2014

Pagination : 109-122

ISSN : 1265-1354

Référence électronique

Olivier Bara, «1814-1815 : construction dramatique des événements et modes théâtraux de symbolisation du présent », Revue d'histoire du XIXe siècle [En ligne], 49 | 2014, mis en ligne le 01 décembre 2014, consulté le 01 mai 2019. URL : http://journals.openedition.org/rh19/4750 ; DOI : 10.4000/rh19.4750 


\section{OLIVIER BARA}

\section{4-1815 : construction dramatique des événements et modes théâtraux de symbolisation du présent}

Les événements sont si variés, si importants, si solennels, la nation tout entière donne maintenant un spectacle si intéressant à l'univers, qu'il ne m'est guère possible de suivre d'ailleurs avec bien de l'exactitude les nouveautés qui se succèdent tous les jours sur nos théâtres. Il se passe sur le Grand Théâtre du Monde des nouveautés trop dignes de l'attention publique pour lui permettre de se laisser distraire par l'analyse d'un vaudeville. Et celles-là, quelle plume pourra jamais les décrire ${ }^{1}$ !

Dans le contexte d'accélération du temps et de diffraction des temporalités propre aux années 1814-1815, une rupture semble s'instaurer entre scène du monde et scènes de théâtre. Ces dernières peineraient à saisir dans le miroir dramatique les facettes changeantes d'une actualité éclatée, plus digne d'attention et d'intérêt que des spectacles dramatiques rendus soudain dérisoires. Pourtant, 1814-1815 ne produit pas que des vaudevilles divertissants, détachés de toute actualité, dénués de toute urgence : avec ses quatre régimes différents, la période se caractérise par la multiplication des œuvres de circonstance en prise directe avec le présent immédiat, œuvres chargées non seulement d'accompagner les retours successifs des puissants, mais de renouer la chaîne brisée du temps et de transmuer les mouvances du réel en réalité stable et lisible. Loin de ne proposer que des plaisirs vaudevillesques, les théâtres enregistrent ou déplacent les faits politiques, rejouent ou tentent de réparer le présent bouleversé. Une période d'anomie et d'accélération des conflits confère ainsi à la scène une fonction proprement démesurée : rétablir une concordance des temps vécus et fonder, par l'illusion du spectacle, la concorde civile. Pourtant, le théâtre officiel, engagé dans la production thuriféraire, est lui-même traversé entre 1814 et 1815 par une double crise : celle du ralliement aux nouveaux régimes des dramaturges accusés de "girouettisme»; celle de la répétition stérilisante, d'une restauration à l'autre,

1. Charles Nodier, feuilleton du Journal des débats, 5 mai 1814. 
des mêmes enthousiasmes concertés. Le "rêve d'unanimisme» propre aux régimes restaurés, ce "rêve de l'instrumentalisation du théâtre au service de [la] concorde politique et sociale ${ }^{2}$ porté par les pièces de circonstance, se heurterait à la réalité d'un esprit public en pleine constitution.

On a voulu se concentrer ici sur la production des pièces de circonstance, œuvres éphémères créées dans l'urgence de l'événement et engagées avec lui dans un rapport spéculaire fondé sur la transposition dramatique immédiate. La définition de ce sous-genre théâtral possède ses limites : toute œuvre représentée dans la période est susceptible de recevoir provisoirement un sens nouveau, adapté aux événements. Elle se fait œuvre de circonstance en contexte, par simple effet de réception : «Il suffisait que l'action roulât sur le retour d'un prince dépouillé, exilé, sur le rétablissement du pouvoir légitime» commente l'historien du théâtre Théodore Muret $^{3}$. Même les pièces nouvelles, conçues à l'origine hors de toute célébration programmée, se trouvent intégrées dans un régime de signification commandé par l'intensité du moment politique ${ }^{4}$. Aussi adoptera-t-on une définition nécessairement ouverte de la pièce de circonstance en se fondant principalement sur la date de représentation et son inscription dans un calendrier politique, festif ou commémoratif, en l'occurrence les périodes comprises entre mi-avril et fin août 1814, mars et juin 1815, début juillet et fin août $1815^{5}$. La méthode ici adoptée consiste à partir du repérage des œuvres, réalisé grâce au dépouillement systématique des annonces de spectacles et des comptes rendus du Journal des débats, et des pièces imprimées en 1814 et 1815 répertoriées sur le catalogue général de la BnF. Dans la limite du présent article, l'étude de réception, à partir des rapports de la police des théâtres, a été écartée $e^{6}$ de même que la prise en compte, par delà les œuvres dramatiques, de la théâtralisation de la vie et du temps

2. Corinne Legoy, «Sous la plume du pouvoir, le public de théâtre entre 1815 et 1830 : l'embarrassant miroir d'une nation souveraine", Parlement[s]. Revue d'histoire politique, 2012/3, n ${ }^{\circ} \mathrm{HS}$ 8, «Scènes politiques", Paris, L'Harmattan, p. 95-108.

3. Théodore Muret, L'Histoire par le théatre, 1789-1851, 2e Série, "La Restauration", Paris, Amyot, 1865, p. 21. Muret (1808-1866) est historien, journaliste, chansonnier, revuiste, dramaturge (co-auteur avec Ludovic Halévy, en 1857, du drame Michel Cervantès). Il fit partie des critiques "libéraux navrés» par les audaces du Roi s'amuse de Victor Hugo (Odile Krakovitch, Hugo censuré. La liberté au théâtre au XIX siècle, Paris, Calmann-Lévy, 1985, p. 51). Son ouvrage L'Histoire par le théatre, s'il doit toujours être lu en contexte et confronté à d'autres sources, demeure une mine de renseignements sur l'accompagnement de la vie politique par les spectacles.

4. Tel est le cas du mélodrame historique de René-Charles Guilbert de Pixerécourt, Charles-leTéméraire ou le Siège de Nancy. Composé à la fin de l'Empire, l'ouvrage a été refusé par la censure en août 1813 et n'est créée, au Théâtre de la Gaité, que le 26 octobre 1814. En contexte, la figure (obligée) du traître de mélodrame, ici revêtu du costume historique, ne peut que renvoyer métaphoriquement à Napoléon. La pièce sera suspendue pendant les Cent Jours avant d'être reprise le 4 août 1815, et jouée notamment le 25 de ce mois, jour de la fête du roi : elle devient pour un jour œuvre de circonstance. Je remercie Barbara T. Cooper qui m’a transmis les informations concernant Charles-le-Téméraire de Pixerécourt et renvoie à son article (à paraître aux Classiques Garnier) sur cette pièce.

5. Nous n'avons pas, dans l'espace restreint de l'article, intégré les pièces jouées dans les moments d'incertitude politique compris entre ces périodes, non liées à la célébration directe de quelque "circonstance" publique.

6. Nous renvoyons sur ce point à l'article, déjà cité, de Corinne Legoy. 
politiques 7 . Nous avons préféré opérer une lecture systématique des pièces de circonstance, afin de cerner, sous les discours obligés, les (dis)continuités dans les modes dramatiques de représentation symbolique du présent.

\section{Discontinuité politique, CONTINUITÉ DRAMatique?}

\section{Première Restauration}

Les pièces de circonstance créées à l'occasion de la première Restauration sont chronologiquement concentrées entre mi-avril et fin août 1814. Les premiers ouvrages, composés dès les premiers jours d'avril, sont joués entre l'entrée du comte d'Artois dans Paris, le 12 avril, et l'arrivée de Louis XVIII dans la capitale le 3 mai.

Pièces de circonstance créées à Paris (avril-août 1814):

- Les Clefs de Paris ou le Dessert d'Henri IV, «trait historique en vaudeville», par Théaulon de Lambert et Dartois de Bournonville, Théâtre du Vaudeville, 20 avril 1814.

- Le Souper d'Henri IV ou la Dinde en pal, "comédie en un acte et en vaudevilles", de Balisson de Rougemont, Théâtre des Variétés, 23 avril 1814.

- Henri IV et D'Aubigné, "comédie en trois actes et en prose», de Balisson de Rougemont et René Perrin, Théâtre de l'Odéon, 28 avril 1814.

- Les Héritiers Michau ou le Moulin de Lieursain, opéra-comique en un acte, de Planard, musique de Bochsa, Opéra-Comique, 30 avril 1814.

- L'Entrée de Henri IV à Paris, Cirque de Franconi [début mai 1814? auteurs non identifiés $]^{8}$.

- Le Retour des Lys, "à-propos en un acte et en vaudevilles, à l'occasion de l'entrée de Sa Majesté Louis XVIII à Paris», par Désaugiers et Gentil, Théâtre des Variétés, 3 mai 1814.

- Henri IV ou la Prise de Paris, mélodrame historique en 3 actes de Boirie, Léopold [Chandezon] et *** [Jean-Baptiste Dubois], Théâtre de la Gaîté, 4 mai $1814^{9}$.

- Vive la paix! ou le Retour au village, «impromptu en un acte, mêlé de chants et de danses ", par Coupart et Varez, divertissement de Millot, Théâtre de l'Ambigu-Comique, 4 mai 1814.

- Les Béarnais ou Henri IV en voyage, comédie en un acte mêlée de chants, par Sewrin, musique de Kreutzer et Boieldieu, Opéra-Comique, 21 mai 1814.

7. Voir Sheryl Kroen, Politics and Theatre. The Crisis of Legitimacy in Restoration France 18151830, Berkeley, University of California Press, 2000.

8. Ce spectacle du Cirque Franconi apparaît sur le programme des théâtres donné par le Journal des débats (feuilleton du 2 mai 1814).

9. Sur cette pièce, voir Barbara T. Cooper, 'History, Politics and Melodrama in Boirie's Henri IV, ou la Prise de Paris', Nineteenth-Century French Studies, 1983, vol. 11, nº 3-4, p. 247-267. 
- L'Île de l'espérance ou le Songe réalisé, "pièce allégorique en un acte mêlée de vaudevilles, à l'occasion de la paix générale", de Désaugiers, Gentil et Brazier, Théâtre des Variétés, 6 juin 1814.

- La Route de Paris ou les Allants et les Venants, "tableau épisodique en un acte, en vaudevilles", par Théaulon de Lambert et [Armand] Dartois de Bournonville, Théâtre du Vaudeville, 30 juillet 1814.

- Le Sabre de bois ou la Revue du roi, "comédie en 1 acte, mêlée de vaudevilles», de Balisson de Rougemont, Théâtre de la Gaîté, 22 août 1814.

- Pélage ou le Roi et la Paix, opéra en deux actes, par Étienne de Jouy, musique de Spontini, ballets et divertissement de Gardel, Académie Royale de musique, 23 août 1814 .

- La Jeunesse de Henri IV ou la Clairière béarnaise, "comédie en un acte mêlée de couplets ", comédie en un acte, par Merle, Brazier et Ourry, Théâtre des Variétés, 24 août $1814^{10}$.

Les théâtres secondaires sont toujours plus prompts à réagir à l'actualité que les théâtres principaux : les Vaudevilles et les Variétés devancent de quelques jours l'Odéon et l'Opéra-Comique, dont la pièce Les Héritiers Michau parvient à s'imposer à l'affiche pour 41 représentations ${ }^{11}$, là où les autres pièces relèvent du spectacle éphémère. Moins immédiatement réactives, tenues par des exigences artistiques supérieures à celles du vaudeville, les scènes principales misent sur des reprises susceptibles de faire écho à l'actualité. On remet en scène des pièces associées à la gloire monarchique et à l'Ancien Régime, élevées à une force de symbolisation renouvelée par le contexte. Ainsi de La Bataille d'Ivry de Durosoy et Martini (1774), tragédie lyrique créée à l'occasion de l'accession au trône de Louis XVI et remise à l'affiche par l'Opéra-Comique dès le 23 avril $1814^{12}$. Le Théâtre-Français affiche Héraclius de Corneille, ouvrage qui ne pouvait "pas manqu[er] de plaire dans les circonstances où nous sommes, n'eût-il offert au public que le rétablissement d'un prince de sang royal sur le trône de son père, et le châtiment d'un tyran ${ }^{13}$. Le Triomphe de Trajan d'Esménard et Persuis, tragédie lyrique créée à l'Opéra en 1807 pour célébrer les victoires impériales, est remanié par Pierre-Ange Vieillard pour être joué à la mi-mai devant les

10. S’ajoutent les pièces imprimées mais (vraisemblablement) non jouées: La Levée en masse, "comédie en un acte mêlée de vaudevilles», par C. J. M. Millin, Paris, "chez les libraires du PalaisRoyal", [juin] 1814; Le Retour du conscrit ou le Rétablissement de Louis XVIII sur le trône de ses ancêtres, comédie en 3 actes d'Alexandre Delannoy, Paris, 1814; Le Siège et la Réduction de Paris ou le Retour de la France à son roi, "pièce historique en 3 actes et en prose", sans nom d'auteur, Paris, Patris, 1814.

11. Par comparaison, Les Béarnais ou Henri IV en voyage, de Sewrin, Kreutzer et Boieldieu, créé à l'Opéra-Comique le 21 mai 1814, n'est joué que sept fois. Je me fonde sur les chiffres établis dans mon ouvrage Le Théâtre de l'Opéra-Comique sous la Restauration. Enquête autour d'un genre moyen, Hildesheim, Georg Olms Verlag, 2001. Sur les pièces de circonstance créées à l'Opéra-Comique, voir en particulier p. 212-233.

12. La Bataille d'Ivry est donnée six fois en 1814 et deux fois en 1815 avant de disparaître du répertoire de l'Opéra-Comique.

13. Charles Nodier, feuilleton du Journal des débats, 23 mai 1814. 
souverains étrangers ${ }^{14}$. On exhume aussi des cartons, au Théâtre-Français, les pièces jusqu'alors interdites par la censure impériale, telle la tragédie Les États de Blois de Raynouard, créée le 31 mai 1814 avec Talma en duc de Guise. La pièce est riche de possibles allusions à l'actualité du moment : «celles qu'offre la pièce de Raynouard sont nombreuses, frappantes, et ont été avidement saisies. On a applaudi avec enthousiasme à tous les vers qui rappelaient une circonstance de nos malheurs récents ou le sentiment du bonheur qui va leur succéder. Quelques traits qui peignent le Henri IV de nos aïeux, et dans lesquels il est impossible de ne pas reconnaittre celui de nos jours, ont excité une espèce d'ivresse. ${ }^{15}$ Entre les œuvres nouvelles inscrivant en direct la rupture politique dans la vie collective, les œuvres anciennes redécouvertes dans leurs nouvelles significations, les œuvres arrachées à l'interdiction impériale, s'élabore un régime temporel complexe, où la rupture opérée dans le présent se trouve à la fois célébrée et compensée par des continuités rétablies à l'échelle du répertoire et de la mémoire des spectateurs.

Dans la production des pièces de circonstance, un pic de créativité s'observe au moment de l'entrée à Paris de Louis XVIII le 3 mai. Les théâtres secondaires renouvellent pour l'occasion leur affiche les 3 et 4 mai. S'adjoint à eux un spectacle de curiosités donné par le cirque Franconi, Le Retour de Henri IV à Paris, titre métaphoriquement transparent. L'ensemble des théâtres parisiens se trouve ainsi progressivement mobilisé : chaque directeur se doit de présenter au moins une œuvre célébrant la circonstance ${ }^{16}$. La stabilisation du régime trouve encore un accompagnement théâtral : la paix signée avec les puissances alliées, le 30 mai, inspire L'Île de l'espérance ou le Songe réalisé aux Variétés le 6 juin. L'approche de la saint Louis (25 août, bientôt décrétée fête nationale ${ }^{17}$ ) entraîne une nouvelle efflorescence de pièces à la Gaîté et aux Variétés comme à l'Opéra. L'activité théâtrale circonstancielle se poursuit au-delà de l'été $1814^{18}$ mais de façon sporadique, hors de la scansion des événements fondateurs du nouveau régime. La dernière pièce liée à l'actualité de la première Restauration est Turenne ou un trait de modestie, vaudeville historique en un acte d'Achille Dartois de Bournonville et Fulgence, créé au Théâtre du Vaudeville de la rue de Chartres, le 23 février 1815, une semaine avant le débarquement de Napoléon à Golfe Juan. Les pièces de cir-

14. Voir Le Triomphe de Trajan, tragédie lyrique en 3 actes, nouvelle édition avec des changements et des corrections par Pierre-Ange Vieillard, Paris, Ballard, 1814.

15. Charles Nodier, feuilleton du Journal des débats, 2 juin 1814.

16. Seul le Théâtre-Italien de Paris ne crée pas d'œuvre de circonstance. Toutefois, lors du concert vocal et instrumental du 11 mai 1814, Drouet "de la musique particulière de S.M. le Roi » joue Vive Henri IV, fantaisie pour la flûte composée par lui-même. Le 23 mai, est joué «le God save the King des Français, paroles de M. le chevalier de Piis, arrangé à grand orchestre par M. Tadolini, couplets chantés par M. Génot, élève du Conservatoire, avec les chœurs». Voir Jean Mongrédien, Le Théâtre-Italien de Paris 1801-1831. Chronologie et documents, Lyon, Symétrie \& Palazetto Bru Zane, coll. «Perpetuum mobile», 2008, vol. III, p. 510 et 521.

17. Voir Francis Démier, La France de la Restauration (1814-1830). Limpossible retour du passé, "folio histoire», Paris, Gallimard, 2012, p. 176.

18. Par exemple, Le Grenadier de Louis XV ou le Lendemain de Fontenoy, pièce en un acte mêlée de couplets de Jean-Baptiste Dubois, créée le 27 décembre 1814 au Théâtre de la Gaîté. 
constance dessinent parfois une histoire chimérique, qu'un présent chaotique rend vite à son inanité ${ }^{19}$.

\section{Cent-Jours}

"Les pièces politiques de 1814 n'avaient pas encore entièrement quitté les affiches, quand arriva le brusque intermède des Cent-Jours; mais ce retour passager de l'Empire n'a laissé aucune œuvre théâtrale qui l'ait célébré. » ${ }^{20}$ L'auteur de ce constat, Théodore Muret, en énonce aussitôt la cause : "Une préoccupation inquiète pesait sur les esprits, et les tenait dans l'attente fiévreuse du lendemain. Les plus intrépides courtisans dramatiques de la circonstance restèrent sur la réserve, sentant, comme les directeurs, que ce n'était pas la peine de se mettre en frais ${ }^{21}$.» De fait, aucune œuvre ne semble avoir accompagné directement le retour de Napoléon. Un seul titre a été découvert : Le Retour d'Arlequin ou l'Heureux Dénouement, "vaudeville en un acte par MM. Thomas et Maillot», refusé par les théâtres. La pièce imprimée contient une dédicace "à Sa Majesté l'Impératrice "22. Les manifestations favorables aux Cent Jours sont essentiellement des couplets chantés sur les théâtres, comme ceux de Coupé Saint-Donat et Lélu interprétés le 25 mars 1815 l'Opéra-Comique : "Le Retour, chant national ${ }^{23}$. Les signes de ralliement à l'empereur peuvent être aussi individuels et symboliques, tel le bouquet de violettes porté par Melle Mars en signe d'amitié. Elle sera contrainte par une partie du public, aux premiers jours de la seconde Restauration, de crier sur scène «Vive le Roi!» ${ }^{24}$. Cela semble confirmer l'analyse de Francis Démier, selon lequel «le rétablissement de la monarchie avait pu avoir, en 1814 , les teintes d'un certain consensus imposé par la lassitude d'une France épuisée; en 1815 , le retour du roi s'accompagne d'une vague brutale de ven-

19. Dans les trois premiers mois de la Restauration, la province propose, dans les principales villes, des pièces de circonstance. La Bonne-Nouvelle ou l'Heureuse journée, comédie-vaudeville en un acte, est «faite à l'occasion de la Paix générale» par Victor Mangin père, jouée au Grand Théâtre de Nantes le 17 mai 1814, deux semaines après l'entrée du roi à Paris. Une durée équivalente sépare la signature de la paix et la représentation au Théâtre de Dijon, le 19 juin 1814, de La Pacification générale ou l'Hôtellerie de Calais, "opéra-vaudeville en un acte», par Chambelland. La veille de "la Fête de Sa Majesté Louis XVIII", La Fête de famille, "opéra-vaudeville-impromptu en un acte et en vers libres, paroles de MM. De Gay-de-La-Tour et E. Lepeintre», est créé sur le Théâtre de la Ville de Versailles. D'autres pièces de circonstance sont éditées en province, sans qu'il soit toujours possible d'établir si elles ont été jouées : Le Premier Avril 1814 ou le Retour des Bourbons, comédie-vaudeville, par L.B.*** et P.S.***, Montpellier, sans nom d'éditeur, 1814, L'Espérance réalisée, opéra-vaudeville en un acte, "à l'occasion de l'heureux avènement de Louis XVIII au Trône», par Alexandre Marie, Toulouse, sans nom d'éditeur ni date.

20. Théodore Muret, L'Histoire par le théâtre, op. cit., 2e Série, p. 33.

21. Ibidem Et l'auteur d'ajouter : "Cette stérilité même est significative, pour peindre la situation, et les théâtres en disent ici beaucoup, précisément parce qu'ils ne disent rien.»

22. Le Retour d'Arlequin ou l'Heureux Dénouement, "vaudeville en un acte par MM. Thomas et Maillot», sans lieu ni date d'édition.

23. «Le Retour, chant national, exécuté à grand orchestre et avec les chœurs, sur le théâtre impérial de l'Opéra-Comique, le 25 mars 1815, paroles de M. le Chevalier Coupé de Saint Donat, musique de M. Lélu ", partition imprimée : Paris, Boulevard des Italiens, ${ }^{\circ} 8$, [1815], Bibliothèque nationale de France, Département de la musique, Vm7-16971.

24. Voir Francis Démier, La France de la Restauration, op. cit., p. 126. 
geances et de règlements de compte qui hypothèque durablement un accord profond entre les Français. ${ }^{25}$ Pourtant, la production thuriféraire de pièces de circonstance installe dans l'univers symbolique de l'été 1815 une toute autre temporalité, faite de réparations, de continuité et de concorde civile, dans un grand fantasme (partagé?) de fin de l'histoire.

\section{Seconde Restauration}

L'entrée de Louis XVIII à Paris, le 8 juillet, deux jours après celle des Prussiens, déclenche bientôt de nouvelles œuvres de circonstance concentrées entre le 16 juillet et la saint Louis, à la fin du mois d'août.

Pièces de circonstance créées à Paris (juillet-août 1815):

- Une fête de famille ou Spectacle demandé, "divertissement impromptu en un acte, mêlé de couplets, à l'occasion du retour du roi ", d'Henri Dupin et Delestre Poirson, Théâtre de la Porte Saint-Martin, 16 juillet 1815.

- L'Écharpe blanche, "fait historique en un acte mêlé de couplets", d'Henri Dupin, Vaudeville, 17 juillet 1815.

- L'Heureux Retour, ballet en un acte, par L.J. Milon, divertissement de Gardel, musique arrangée par Persuis, Berton et Kreutzer, Académie Royale de Musique, 25 juillet 1815.

- Voilà notre bouquet! ou le Cabinet littéraire, «impromptu-vaudeville en un acte" de Coupart et Varez, Théâtre de l'Ambigu-Comique, 31 juillet 1815.

- Une soirée des Tuileries ou l'Été de 1815, "divertissement en un acte et en prose, mêlé de chants, par Georges Duval ", Théâtre de l'Odéon, $1^{\text {er }}$ août 1815.

- Le Roi et la Ligue ou la Ville assiégée, opéra-comique en 2 actes, de Théaulon et Armand Dartois, musique de Bochsa, Opéra-Comique, 22 août 1815.

- Le Bouquet du Roi ou Le Marché aux fleurs, "à propos en un acte, mêlé de vaudevilles» de Désaugiers et Gentil, Théâtre des Variétés, 23 août 1815.

Moins nombreux que lors de la première Restauration, les ouvrages de circonstance obéissent aux mêmes règles de fait : légère antériorité et prédominance des théâtres secondaires sur les scènes principales - le Théâtre-Français se contente de reprendre La Partie de chasse de Henri IV de Charles Collé. Le bégaiement de l'histoire permet de rejouer les pièces de circonstance créées lors de la première Restauration, au risque d'épuiser leur matériau symbolique et leur puissance d'entraînement : Les Héritiers Michau à l'OpéraComique, Le Souper de Henri IV aux Variétés. La province suit encore le mouvement parisien. Au Grand Théâtre de Bordeaux, La Ville de Mirmont donne Childéric 1 $^{e r}$, tragédie en trois actes, le 28 août 1815. L'«Avertisse-

25. Idem, p. 131. 
ment» de l'auteur précise : «je n'ai voulu écrire que pour la circonstance, et n'ai pas prétendu travailler pour la postérité » ${ }^{26}$. Egidius, centurion romain, est l'usurpateur du trône de France tandis que Childéric, père de Clovis, est proscrit jusqu'à l'heureux dénouement : la métaphore historique est limpide.

\section{LES AUTEURS : DES «GIROUETTES »?}

Les pièces de circonstance de la première Restauration s'élaborent dans une apparente spontanéité, gage de leur sincérité. Du moins est-ce le mythe entretenu par une partie de la presse - à moins qu'il ne faille lire quelque ironie par antiphrase entre les lignes :

«Nous ne sommes pas éloignés du temps où un hommage poétique était une spéculation comme une autre, qui devait rapporter en proportion de la bassesse, et non en proportion du talent, et qui donnait à la fortune tous les droits qu'elle ôtait à l'honneur. Alors, on jetait des poèmes dans le commerce, on plaçait des odes à intérêt, et on faisait d'un vaudeville une espèce de lettre de change payable à vue. Il n'en est pas de même aujourd'hui. Un gouvernement qui est fondé sur les droits les plus touchants et les plus sacrés, n’a plus besoin de payer la flatterie, puisqu'il est presque obligé de réprimer le sentiment ${ }^{27}$.

Le propos émollient de Nodier s'inscrit dans l'esprit de la première Restauration, en quête d'une réconciliation rapide de la nation. La dénonciation de l'insincérité des auteurs s'installe en revanche dans le discours public lors de la seconde Restauration. Plusieurs auteurs des pièces de circonstance de 1814-1815 sont intégrés au Dictionnaire des Girouettes paru en juillet 1815 et réédité deux fois jusqu'à la fin de l'année ${ }^{28}$. Antoine-Marie Coupart, auteur avec Varez de Vive la paix! en 1814 et Voilà notre bouquet! en 1815, après avoir offert des vers pour la naissance du roi de Rome, est ironiquement présenté comme "employé au ministère de la police, bureau des théâtres, sous l'empereur, le roi et l'empereur ${ }^{29}$. Marc-Antoine Désaugiers, auteur avec Michel-Joseph Gentil de Chavagnac du Retour des Lys et de L'Tle de l'espérance, se serait depuis longtemps adapté aux changements de régime :

26. La Ville de Mirmont, Childéric Ier, Bordeaux, Lavigne jeune, 1815. Je remercie Maurizio Melai d'avoir attiré mon attention sur cette œuvre.

27. Charles Nodier, feuilleton du Journal des débats, 11 mai 1814.

28. Voir Pierre Serna, «La bataille des Girouettes... Du bon usage du changement d'opinion

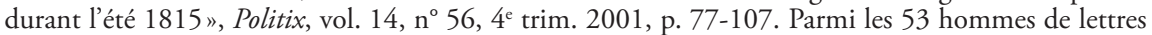
figurant au nombre des Girouettes, 9 sont dramaturges et 14 chansonniers ou vaudevillistes, ce qui signale l' «importance de ces intermédiaires culturels dans la construction du paysage politique français de la Restauration" (ibidem, p. 96).

29. Dictionnaire des Girouettes, ou nos contemporains peints d'après eux-mêmes, Paris, Alexis Eymery, 1815 ( $2^{e}$ édition), p. 107. Coupart, né en 1780, a été nommé en 1799 au ministère de la Police, dans l'administration des journaux et des théâtres. D'après Corinne Legoy, L'Enthousiasme désenchanté. Éloge du pouvoir sous la Restauration, Paris, Société des Études robespierristes, 2010, p. 30. 
"Avant de célébrer l'empereur, l'impératrice, le roi de Rome, le roi de France et de Navarre, M. Désaugiers, suivant la maxime de Franklin, avait chanté Dieu et la liberté. " ${ }^{30}$ Michel-Nicolas Balisson de Rougemont, "hyperbolique thuriféraire de 1810 et $1811 »^{31}$, se convertit à la célébration monarchique. Sa production d'éloges et de pièces de circonstance, de l'Empire à la Seconde Restauration, des Stances sur le mariage de S.M.I. et R. Napoléon avec S.A. l'Archiduchesse Marie-Louise au Chansonnier des Bourbons, illustrerait son opportunisme sans scrupule ${ }^{32}$. Étienne de Jouy, librettiste de l'opéra Pélage ou le Roi et la Paix, opère quant à lui deux ralliements successifs à la monarchie, lors des deux restaurations, alors qu'il avait retrouvé un poste officiel sous l'Empire pendant les Cent-Jours ${ }^{33}$. En revanche, certains dramaturges et poètes apparaissent comme d'authentiques et durables monarchistes, tels les frères Dartois (ou d'Artois de Bournonville), Armand, Achille et Théodore ${ }^{34}$. Toute pièce de circonstance ne relève donc pas de l'écriture insincère et stipendiée. De même, il existe des degrés de "girouettisme», selon le nombre de retournements de position successifs : "Il y a girouette et girouette» reconnaîton dans l'avant-propos du Dictionnaire des Girouettes ${ }^{35}$.

30. Dictionnaire des Girouettes, op. cit., p. 131. Michel-Joseph Gentil de Chavagnac a, de son côté, composé avec Balisson de Rougemont Les Fêtes françaises ou Paris en miniature pour le mariage de l'empereur (Variétés, 1810), ainsi que, seul, La Bonne nouvelle ou le premier arrivé, pour la naissance du roi de Rome (Variétés, 1811). Même parcours pour Maurice Ourry, auteur de La Double Fête pour la fête de Napoléon (Variétés, 1810), coauteur de La Jeunesse d'Henri IV sous la première Restauration (Variétés, 1814).

31. Théodore Muret, L'Histoire par le théâtre, op. cit., p. 27.

32. Michel-Nicolas Balisson de Rougemont (1781-1840) offre en effet une production thuriféraire variée, réorientée au gré des changements de régime. Avant et après les pièces jouées en 1814 et 1815, il compose : Le Retour du héros, poème [1805]; La Paix, divertissement en vaudevilles, Théâtre des Variétés, 23 octobre 1809; La Fête impromptue, divertissement en un acte, mêlé de vaudevilles, à l'occasion de la fête de Leurs Majestés, Théâtre de l'Impératrice, 14 août 1810; Le Mariage de Charlemagne, tableau historique en un acte et en vers, Théâtre de l'Impératrice, 14 juin 1810 (comprenant des allusions à l'Empereur); L'Olympe, Rome, Paris et Vienne, scènes épisodiques, pour la naissance du roi de Rome, 1811; Le Chansonnier des Bourbons (avec J.A. Jacquelin), dédié à S.A.S. Madame la duchesse douairière d'Orléans, 1815 (épître dédicatoire : "On chante par amour des Princes bienfaisants;/ Les Bourbons n'aiment point un mercenaire encens [...]»); La Saint-Louis villageoise, comédie en un acte, Théâtre des Variétés, 24 août 1816; La Fête de Henri IV, comédie en un acte et en vers libres, Théâtre-Français, 23 août 1816; Les Deux Mariages, à-propos à l'occasion du mariage du duc de Berry, Paris, Théâtre des Variétés, 15 juin 1816; L'Espagne délivrée, dithyrambe à Son Altesse Royale Monseigneur, duc d'Angoulême [1823]; Ode sur la mort de Louis XVIII, 1824.

33. Ses revirements sont assurés par les transformations de son personnage d' "Hermite de la Chaussée d'Antin " créé pour la Gazette de France, devenu Guillaume, cousin de l'Hermite, sous la première Restauration, puis l'Hermite de la Guyane dans Le Mercure de France à partir de juillet 1815. Voir, dans ce numéro, "Écrire la discontinuité, et en mourir: L'Hermite de la Chaussée d'Antin en avril 1814 ", document présenté par Judith Lyon-Caen, et Judith Lyon-Caen, "Étienne de Jouy", dans Dominique Kalifa, Philippe Régnier, Marie-Ėve Thérenty, Alain Vaillant [dir.], La Civilisation du journal. Histoire culturelle et littéraire de la presse française au XIX é siècle, Paris, Nouveau monde éditions, 2011, p. 1101-1105.

34. "Théaulon et Dartois, deux noms fraternellement unis dès leurs débuts dans la carrière, furent par excellence les auteurs royalistes de la première et de la seconde Restauration. » Dictionnaire des Girouettes, op. cit., p. 245. De fait, on retrouve leur nom associé aux pièces de circonstance de la seconde Restauration, notamment à l'Opéra-Comique (voir mon ouvrage, Le Théâtre de l'OpéraComique sous la Restauration, op. cit., p. 217 et 221).

35. Dictionnaire des Girouettes, op. cit., p. xi. 
Ces dramaturges, vaudevillistes, chansonniers seraient contraints à un "conformisme servile à l'égard de tous ceux, possédants et dirigeants, qui peuvent par leurs commandes, assurer leur survie économique ${ }^{36}$. Une lettre autographe du compositeur Gaspare Spontini à «Monseigneur» éclaire la position de l'artiste face au pouvoir : «Le retour à Paris à jamais heureux de Votre Altesse Royale en France redouble le désir que j'ai de m'y distinguer par des productions estimées et de me dévouer entièrement au service du Roi et de son auguste famille. ", écrit l'auteur de Pélage en préambule. Réclamant la protection de Louis XVIII pour l'obtention de la place de «directeur de la musique particulière du Roi et de l'Opera-Buffa et Seria italien", Spontini rappelle : "Quant à mes sentiments pour le Roi, ils sont connus. Le premier d'avril dernier, je travaillais déjà à Pélage ou le Roi de la paix [sic], qu'on a donné au Grand Opéra et dont Sa Majesté a daigné agréer la dédicace. ${ }^{37} \mathrm{La}$ lettre révèle comment des politisations factices servent des intérêts personnels dans la lutte entre les hommes de lettres ou entre les musiciens, dans un moment de désorganisation profonde du champ artistique. Le corpus des pièces de circonstance en 1814-1815 montre que quelques auteurs parviennent à s'imposer durablement dans ce sous-genre dramatique : «[...] Brazier, Rougemont, Gentil de Chavagnac, Théaulon de Lambert, Désaugiers et Coupart monopolisent [...] la production dramatique et chansonnière contribuant à la célébration du pouvoir dans la capitale. " ${ }^{38}$

Il ne faut donc pas voir derrière la production de pièces de circonstance en 1814-1815 quelque propagande concertée et payée. Si l'on en croit Théodore Muret, la rétribution des poètes de circonstance baisse drastiquement entre Empire et Restauration : «L'Empire allouait mille francs aux auteurs pour chacune de ses pièces de circonstance : la Restauration réduisit le chiffre à cinq cents francs, et même ensuite elle ne donne plus qu'une médaille qui en valait environ deux cents. Pour un peu qu'une œuvre de ce genre eût quelques représentations, les droits d'auteur la payaient bien assez »39. L'impulsion vient d'abord des directeurs de théâtre, soumis à l'État dans le système des privilèges; les pièces de circonstance peuvent être décidées en concertation avec le ministère de la Maison du Roi et sont contrôlées par la censure préalable. On est proche du mode d'incitation étudiée par Corinne Legoy concernant la publication d'éloges en vers : «La monarchie restaurée ne fait, au mieux, que favoriser la production et la diffusion par le biais de pensions, de gratifications ou de souscriptions. Ce faisant, la pratique thuriféraire n'est liée au mécénat d'État que par les gratifications espérées, dans un système où l'écrivain n'obtient pas encore des revenus suffisants du marché du livre lui-même. $»^{40}$ La période de ruptures que forment les années 1814-

36. Pierre Serna, art. cité, p. 95.

37. Cité par Jean Mongrédien, Le Théatre-Italien de Paris, op. cit., vol. III, p. 506-507.

38. Corinne Legoy, L'Enthousiasme désenchanté, op. cit., p. 37.

39. Théodore Muret, L'Histoire par le théâtre, op. cit., p. 14-15.

40. Corinne Legoy, L'Enthousiasme désenchanté, op. cit., p. 39. 
1815 révèle la transformation à l'œuvre dans le statut de l'écrivain, pris entre ancien mécénat d'État et affirmation (romantique) de l'autonomie créatrice, portée par l'affirmation de la sincérité littéraire. C'est donc la dénonciation du "girouettisme» qui fait sens, également pour ces raisons avancées par Pierre Serna : «En 1814, fort peu se gaussent des "comportements alternatifs", parce qu'ils peuvent encore souder la société dans la réconciliation et dans une histoire commune des décennies écoulées, même si l'on peut encore la penser anormale. En 1815, la girouette est inventée pour refuser et dénigrer ces comportements immoraux qui, par la recherche du compromis le plus cynique, ne peuvent, maintenant, que diviser davantage les Français, toujours plus désorientés quant à l'avenir ou toujours plus accrochés à leur représentation du passé.» ${ }^{41}$

Le phénomène le plus marquant est bien la rencontre entre le soupçon de palinodie porté par une partie de l'opinion, ou du moins imposé à elle par les discours publics, et le contenu même des pièces de circonstance, célébration d'une fidélité inconditionnelle par delà les aléas historiques. Entre 1814 et 1815 est mis en question l'artifice des enthousiasmes théâtralisés.

\section{Présence efficace et CONTINUité RÊVÉE}

\section{Le présent fait spectacle}

«[L'intrigue] est presque toujours nulle dans ces sortes d'ouvrages. Autrement elle détruirait le véritable intérêt qui est celui de l'heureux événement qu'on célèbre» déclare en préface l'auteur de La Pacification générale ou l'Hô-

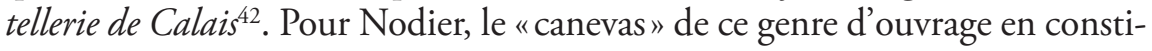
tue la partie faible, la moins importante; le "principal mérite» réside dans le «choix des détails», le «tour des couplets», le «bonheur des allusions $»^{43}$. Les intrigues sont réduites à de simples prétextes tandis que l'action est souvent inexistante, surtout dans les pièces chargées de transposer l'événement immédiat. On verra sur scène maints villages dépeuplés de leurs hommes par les guerres de l'Empire où les femmes attendent le retour des maris ou des prétendants (Le Retour des lys, Vive la paix!) Le schéma admet des variations pathétiques, par exemple lorsque le fiancé est cru mort, avant de reparaitre bien vivant au dénouement (dans le ballet L'Heureux retour). Autres variantes, permettant d'introduire le fait politique à fêter et de l'élever au statut d'événement digne de contemplation et de mémorisation : un père promet la main de sa fille à qui ramènera un lys (Le Retour des lys) ou une simple bonne nouvelle en ces temps de guerre, de conscription et de ruine économique ( $\mathrm{La}$

41. Pierre Serna, art. cité, p. 107.

42. [Claude-Antoine] Chambelland, La Pacification générale ou l'Hôtellerie de Calais, "opéravaudeville en un acte», créé au Théâtre de Dijon le 19 juin 1814, Dijon, De l'imprimerie de Carion, juillet 1814 , p. viii.

43. Charles Nodier, feuilleton du Journal des débats, 5 mai 1814. 
Bonne Nouvelle). La faiblesse même d'une intrigue mal nouée et d'une action faiblement unifiée contribue à conférer à la péripétie ou à la catastrophe une apparence prodigieuse, transformation soudaine, non motivée dramatiquement, de la situation. Dans Vive la paix! de Coupart et Varez, alors que le village est déserté par les hommes, la scène se remplit d'un coup de "tous les garçons du village» tandis qu'on déclame ce récit, conférant une soudaine profondeur de champ à une intrigue domestique : «Les souverains paraissent : la bonté qui se peint dans tous leurs traits, fait disparaitre la crainte; bientôt on se presse, on les entoure, et ces mots consolants échappent à l'un d'eux : Français, je vous apporte la paix et le commerce. ${ }^{44}$ Récit édifiant d'une présence réputée efficace, source d'un ravissement émotionnel.

Les pièces de circonstance organisent la matérialisation scénique, visuelle et sonore, de l'événement contemporain, accédant ainsi à une forme d'hyper-présence et d'omniprésence sur les scènes ${ }^{45}$. Les symboles monarchiques saturent l'espace visuel chargé de fleurs de lys et de drapeaux blancs : jeunes gens de retour au village "un lys à la main, la cocarde blanche et un drapeau orné de fleurs de lys", avant que des «lettres de fleurs portés par cinq personnes se réunissent pour former le nom de LOUIS» (Le Retour des lys $s^{46}$ ); apparition d'un écusson portant l'inscription "vive Louis XVIII", venant remplacer l'affiche d'appel à la conscription (Vive la paix. ${ }^{147}$ ). Une telle occupation de l'espace visuel et sonore de la scène redouble la saturation de l'espace urbain : dans les moments d'incertitude sur la localisation d'une souveraineté incertaine, l'exhibition des symboles du pouvoir dans l'espace public pallie son absence ou sa défaillance ${ }^{48}$. Au théâtre, elle est aussi une réponse à l'impossible présence du roi. Au sein de ces tableaux scéniques de liesse nationale, l'absence de représentation directe du roi est en effet remarquable. Elle assure la concentration des regards vers le point de fuite de la scène : le réel politique au-delà de sa théâtralisation. Ainsi dans Une soirée des Tuileries, les personnages tentent en vain d'apercevoir le monarque, regardant "du côté où est censé être le Roi, et vers lequel tous les personnages en scène ont les yeux fixés ${ }^{49}$. Se déploient dans l'espace visuel et sonore des substituts métaphoriques de Louis XVIII (Henri IV et toutes ses déclinaisons drama-

44. Antoine-Marie Coupart et E. F. Varez, Vive la paix! ou le Retour au village, "impromptu en un acte, mêlé de chants et de danses ", Paris, Maugeret, 1814, scène 3.

45. Je me permets de renvoyer à mon article «Dramaturgies de la souveraineté : entrées royales et pièces de circonstance sous la Restauration", dans Corinne et Éric Saminadayar-Perrin [dir.], Imaginaire et représentations des entrées royales au XIX'e siècle : une sémiologie du pouvoir politique, Presses de l’Université de Saint-Étienne, 2006, p. 41-60.

46. Marc-Antoine Désaugiers et Michel-Joseph Gentil de Chavagnac, Le Retour des lys, "à propos en un acte et en vaudeville", Paris, chez les marchands de nouveautés, 1814, scène 18.

47. Vive la paix!, op. cit., "ballet final».

48. Les emblèmes du pouvoir politique «permettent le marquage de l'espace par une souveraineté théoriquement incontestée, en dépit de la pluralité politique héritée de la Révolution" (Emmanuel Fureix, "Police des signes, ordre et désordre politiques en temps de crise (France, 1814-1816)", article à paraître dans Histoire urbaine (mes remerciements à l'auteur).

49. Georges Duval, Une soirée des Tuileries ou l'Été de 1815, "divertissement en un acte et en prose mêlé de chants ", Paris, Barba, 1815, scène 13. 
tiques $^{50}$ ) ou plus souvent métonymiques, telle la croix de Saint-Louis dévoilée par un personnage à l'annonce de la proclamation du nom de Louis XVIII dans Paris (Le Premier Avril 1814 ${ }^{51}$ ). La représentation accède alors à une valeur performative. Dire, montrer, saturer l'espace, cela fait exister l'événement collectif dans les consciences, les imaginaires et les corps particuliers, appelés à rejoindre la "communauté affective " $^{52}$ des spectateurs-sujets du royaume. Le brouillage du référentiel et du chimérique, la contamination des aléas du réel par la nécessité théâtrale, arrachent le fait à la contingence de l'actualité pour en imposer illusoirement l'absolue, nécessaire et permanente présence.

\section{Lieux communs : retours et ressassements}

"Vive le roi est un mot excellent qui fait battre le cœur de tous les bons Français; mais il ne coûte pas de grands frais d'imagination au poète qui en fait l'âme d'une scène, et qui en tire l'effet d'une situation ou le trait d'un couplet." La remarque de Nodier dans le feuilleton du Journal des débats (8 mai 1814) pointe le caractère nécessairement répétitif des pièces de circonstance. On assiste d'une restauration à l'autre à la même célébration unanimiste d'une fin: des guerres, de la conscription, de la crise commerciale. Le renouveau escompté, proclamé de facto, est actualisé en scène. Ainsi, dans La Route de Paris ou les Allants et les Venants, une aubergiste assiste, comme en une revue, au défilé des clients revenus dans son établissement, le mouvement signifiant et accomplissant le retour de la prospérité. Parallèlement, l'Empereur est rejeté dans un passé révolu, annihilé par la parole accusatrice libérée sur scène ("oppresseur», "Corse inhumain», «usurpateur»). À côté des ouvres montrant l'actualité transformée en histoire présente, la démultiplication des figurations scéniques d'Henri IV dans les pièces historiques est chargée de dire la refondation du caractère national jugé authentique et de légitimer le pouvoir du nouveau roi par la suggestion spectaculaire de la continuité dynastique.

Le théâtre entreprend dès lors de renouer, du moins dans les imaginaires, la chaîne des temps. Dans Les Héritiers Michau, à l'Opéra-Comique, les héritiers ont conservé la maison et les meubles qui accueillirent autrefois Henri IV; à la proclamation du retour des Bourbons, les gardiens de la mémoire sont tout prêts à recevoir le nouveau roi, descendant du monarque hébergé par leurs aïeux. Un couplet de La Pacification générale concentre le fantasme à entretenir parmi les spectateurs : "L'honneur revient en France

50. «Dès 1814, Henri IV s'est installé dans le discours et la symbolique politique de la Restauration. La référence à Henri IV fait lien avec le peuple, la vie quotidienne, une certaine simplicité, une vocation protectrice d'un monarque modéré, attentif au bien-être de son royaume. Elle convient peut-être aussi à l'image d'un Louis XVIII qui aimait tant la table.» Francis Démier, $\mathrm{La}$ France de la Restauration, op. cit., p. 180.

51. Le Premier Avril 1814 ou le Retour des Bourbons, op. cit., scène 11.

52. Formule employée par Alain Corbin dans sa préface à Corinne Legoy, L’Enthousiasme désenchanté, op. cit., p. 6. 
comme par le passé / La noble bienfaisance comme par le passé / La sage expérience plus que par le passé; / Et la douce clémence, / Comme par le passé ${ }^{53}$. Comme le suggère la variante du refrain, la temporalité élaborée par les pièces de circonstance est des plus complexes. Les œuvres naissent, après une gestation rapide, d'une actualité disruptive. Elles sont vouées à disparaître très vite avec le fait célébré. Leur régime se confond avec celui de la fête, éphémère, non reproductible. Le temps représenté en leur sein est celui d'un retour à l'âge d'or, délivrant soudainement du passé honni, ouvrant sur un avenir sans devenir dès lors que l'histoire, vécue comme souffrance et perte, est abolie. Fruits d'une discontinuité temporelle, les pièces de circonstance s'élaborent dans la célébration d'une continuité rétablie; elles évacuent toute historicité dans le temps ritualisé de la cérémonie théâtrale. Car il s'agit de créer un "temps affectif " " temporelle en fantasme de réitération légendaire et d'enroulement mythique. Que la réception de telles œuvres repose sur l'adhésion convenue, le désintérêt distant, ou la croyance enthousiaste est malaisé à établir. L'on peut gager que la concomitance de ces trois régimes de réception fondait le plaisir particulier des spectateurs appelés à figurer, le temps d'une représentation, un corps social fusionnel. Plus important pour l'historien est ce que disent en creux la répétition voire le ressassement des œuvres de circonstance, entre première et seconde Restauration : une désorientation grandissante des spectateurs, invités par les thuriféraires du nouveau régime à des célébrations trop théâtrales pour vraiment rassurer, trop répétitives pour durablement éclairer. C'est en ce sens que l'analyse du théâtre parle à l'historien, l'invitant à appréhender autrement l'événement, à repenser les scansions classiques pour considérer la coexistence complexe, au-delà de la matérialité des faits et de la rhétorique des discours officiels, de représentations symboliques, de réceptions, de paroles et d'actes divergents, et pourtant concomitants.

Olivier Bara est professeur à l'Université Lyon 2, membre du LIRE (UMR 5611)

53. La Pacification générale, op. cit., scène 9.

54. L'expression est de Corinne Legoy dans L'Enthousiasme désenchanté, op. cit., p. 94. 